\title{
COMMISSIONING THE 2.5-MEV RFD LINAC PROTOTYPE*
}

\author{
D.A. Swenson, F.W. Guy, and W.J. Starling \\ Linac Systems, 2167 N. Highway 77, Waxahachie, TX 75165
}

\begin{abstract}
The experience of commissioning a $2.5-\mathrm{MeV}$, "Proof-ofPrinciple" prototype of the new RFD linac structure will be reported. This structure resembles a drift tube linac (DTL) with radio frequency quadrupole (RFQ) focusing incorporated into each drift tube, hence the name, $\underline{\mathrm{R}}$ Focused Drift tube (or RFD) Linac. A prototype of this structure, comprising a $25-\mathrm{keV}$ proton ion source, an Einzel-lens-based LEBT, a 0.65 -m-long RFQ linac to 0.8 $\mathrm{MeV}$, and a 0.35 -m-long RFD linac to $2.5 \mathrm{MeV}$, has been designed, fabricated, assembled, and commissioned in our laboratory. Comparisons will be made between the projected and observed performances of this structure. The effect of this new linac structure on the size, cost and complexity of linac-based systems for practical applications will be described. Plans for the use of this new linac structure to satisfy several scientific, industrial and medical applications will be outlined.
\end{abstract}

\section{DESCRIPTION OF THE PROTOTYPE}

A $2.5-\mathrm{MeV}$ prototype of a "Compact $12-\mathrm{MeV}$ Proton Linac for PET Isotope Production" is under construction at Linac Systems. This unit will serve as the "proof of principle" for the revolutionary new Rf Focused Drift tube (RFD) linac structure. This structure resembles a drift tube linac (DTL) with radio frequency quadrupole (RFQ) focusing incorporated into each "drift tube". Both the prototype and the production unit will operate at 600 MHz. The prototype comprises a $25-\mathrm{keV}$ proton ion source, an dual einzel-lens-based LEBT, a 0.65-m-long RFQ linac to $0.8 \mathrm{MeV}$, and a 0.35 -m-long RFD linac to $2.5 \mathrm{MeV}$. The two linac structures will be resonantly coupled and powered by a collection of twelve planar triodes. The extreme simplicity of the interface between the two structures contributes to the practicality of this operational test on a limited budget. The entire length of the two linacs, including their interface, is only one meter. The entire assembly will be evacuated by 2 turbomolecular pumps and 1 ion pump. The alignment philosophy is based on precision machining and "hard socket" for installation of the drift tubes. We believe that this new structure will become the structure of choice to follow RFQ linacs in many scientific, medical, and industrial applications.

\section{ION SOURCE AND RFQ LINAC COMMISSIONING}

A prerequisite to testing the performance of the RFD linac structure was the testing of the properties of the $0.8-\mathrm{MeV}$ beam to be injected into the RFD linac structure. This beam originates in the duoplasmatron ion source, passes through the dual Einzel-lens LEBT, and is accelerated to $0.8-\mathrm{MeV}$ by the $600-\mathrm{MHz}$ RFQ linac of the Prototype.

The performance of the Ion source and LEBT was established many months ago by measuring the proton beam current on and through a segmented aperture and falling on a viewing screen. For a $25-\mathrm{kV}$ ion source potential and Einzel-lens potentials near $25 \mathrm{kV}$, a few milliampere proton beam converged so that most of it went through the segmented aperture and formed a small spot at the center of the viewing screen. Some electrostatic steering was available, but seemed not to be required.

As the RFQ linac gets its rf power from the RFD linac via the resonant coupler, there is no provision for exciting the RFQ linac without also exciting the RFD linac structure. In order to confirm the performance of the RFQ linac, independent of the RFD linac, it was necessary to intercept the beam from the RFQ linac inside the RFD linac and to diagnose its properties while the RFD linac structure was excited with rf power. In order to do this, we removed the RFD drift tubes and insert a single, larger diagnostic drift tube fitted with a $0.5-\mathrm{MeV}$ absorber foil and a Faraday cup. Without the foil, there was a steady increase in beam current with rf power level until some maximum current was reached. With the foil, a definite threshold was observed in the rf power level, below which no beam was accelerated. Based on this threshold, estimates of the design excitation of the RFQ linac were established.

\section{RFD LINAC COMMISSIONING}

Following the commissioning of the RFQ linac, the RFD drift tubes were reinstalled and the resonant coupler slot dimensions were adjusted to give the desired ratio rf fields in the two linac structures.

For the first few hours of excitation, the RFD linac structure was hung up in a solid multipactor. Although the twin-bladed stems of the RFD drift tubes are prone to

Supported by the National Institute of Mental Health (NIMH). 
multipactor, it proved possible to break through that phenomenon. However, once through the multipactor regime, we were unable to get much more power into the combined structures. The following problems were observed and fixed:

1) Discrepancies were found in the phase and amplitude of the drive to each of the 12 final power amplifier (FPA) planar triode tubes. The rf drive configuration involves a three-way power splitter at the IPA amplifier and 3 fourway power splitter near the FPA. The four-way power splitters were replaced with a new and improved design.

2) Standing waves in the rf drive configuration resulted in voltages that exceeded the voltage capabilities of the RG316 coax and the SMA connectors that were being used. The configuration was changed to use RG-223/U coax and TNC connectors.

3) The length of the 3-1/8 rigid coax line between the FPA and the RFD linac was designed to be one wavelength ( 0.50 meters). The length of this line is critical to the performance of the rf system. Tests were made with this line both lengthened by $25 \mathrm{~mm}$ and shortened by $50 \mathrm{~mm}$. The shorter option seems to be preferred by the rf power protection circuitry.

4) Some of the rf contacts in the sliding tuners of the rf power system were very difficult to operate. These sliding contacts were reworked.

5) Measurements of the $Q$ of the combined structure indicated that the power required for design excitation was more that we could get from the rf power system. SUPERFISH implies that the RFD should have a stored energy of $2.57 \mathrm{~J}$. Early estimates of the Q (or power requirement) for the RFD indicated a $\mathrm{Q}$ of about 48,000, implying a power requirement of $200 \mathrm{~kW}$. Improved estimates of the stem losses (two-bladed stems for 12 short cells) and the end wall losses (two end walls for a very short tank) have reduced the expected $\mathrm{Q}$ to 37,000 , implying a power requirement of $262 \mathrm{~kW}$. The best measured Q of the RFD (isolated from the RFQ) is about 18,000 , implying a power requirement of $538 \mathrm{~kW}$.

\section{A SEARCH FOR CAVITY $Q$}

At this point, a study was launched to determine why the $\mathrm{Q}$ was so low. Under suspicion were the joints between tank ends and the end walls (spring ring under mild compression), the surface conductivity of the copper plating, and the ion pump port. The coupling slot to the RFQ was closed to eliminate the RFQ from suspicion.

Many attempts were made to improve the $\mathrm{Q}$ by replacing the original spring ring join with joints based on spring rings under higher compression, copper braid covered spring ring, solid aluminum wire and soft silver wire under very high compression. None of these test resulted in much of an increase in the Q of the structure.

Many attempt were made to test the rf conductivity of the end plates by using an open-sided S-band cavity against the planar copper-plated surfaces of the Prototype. These measurements are difficult to make and were inconclusive.

Finally, we removed the RFD drift tubes, leaving a right circular cylindrical tank 376-mm in diameter and 342.26$\mathrm{mm}$ long. Here we have the possibility to compare our measurements with the well-known properties of the $\mathrm{TM}_{010}$ and $\mathrm{TE}_{011}$ modes in a right circular cylinder. The $\mathrm{TE}_{011}$ mode is of particular interest because it involves only circumferential currents, making the field distribution and $Q$ virtually independent of the quality of the electrical contact between the cylindrical tank and the end plates. This mode primarily tests the conductivity of the copperplated cavity surfaces. The $\mathrm{TM}_{010}$ mode is the drift tube linac mode and is dependent on both the quality of the cavity surfaces and the quality of the joints between the cylindrical tank and the end plates.

The frequency of both modes were within one tenth percent of their theoretical values $(610.3 \mathrm{MHz}$ for the $\mathrm{TM}_{010}$ mode and $1061.8 \mathrm{MHz}$ for the $\mathrm{TE}_{011}$ mode). The $\mathrm{Q}$ of the $\mathrm{TE}_{011}$ mode $(59,489)$ was $65 \%$ of the theoretical value of 91,507 . The $\mathrm{Q}$ of the $\mathrm{TM}_{010}$ mode $(16,000)$ was $35 \%$ of the theoretical value of 45,600 . This suggests a problem with both the surface conductivity of the copper plating and the conductivity of the end joints.

The RFD linac tank and end plates have been redesigned to utilize copper Helicoflex seals for both the rf and vacuum seal. The design includes an elastomer seal behind the Helicoflex seal for back-up purposes. The linac tank and end plates will be stripped of copper and replated. This work should be completed by the second week in April.

\section{FINAL COMMISSIONING PLANS}

We expect to continue the commissioning of this prototype as soon as the RFD linac structure is re-plated. The challenge then will be to get enough rf power into the structure, to establish that there is an accelerated beam and to measure the properties of it.

\section{APPLICATIONS FOR THE RFD LINAC}

We expect the RFD linac structure to form the basis of a new family of compact, economical, and reliable linac systems serving a whole host of scientific, medical, and industrial applications. The principal medical applications include the production of short-lived radio-isotopes for the positron-based diagnostic procedures (PET and SPECT), the production of epithermal neutron beams for BNCT, 
and accelerated proton beams for injection into proton synchrotrons to produce the energies required for proton therapy. We propose S-Band versions of the structure to serve as $70-\mathrm{MeV}$ injectors to $200-\mathrm{MeV}$ Disk and Washer (DAW) booster linacs for the proton therapy application.

The principal industrial and military applications include the production of intense thermal neutron beams for Thermal Neutron Analysis (TNA), Thermal Neutron Radiography (TNR), and Nondestructive Testing (NDT). High duty factor RFD linac systems could produce nanosecond bursts of fast neutrons to support Pulsed Fast Neutron Analysis (PFNA).

\section{REFERENCES}

1. D.A. Swenson, "RF-Focused Drift-Tube Linac Structure", 1994 Intern. Linac Conf., Tsukuba, Japan, 1994.

2. D.A. Swenson, "A New Linac Structure for the BNCT Application", Proc. First Intern. Workshop on Accelerator-Based Neutron Sources for BNCT, Jackson Hole, WY, 1994.

3. D.A. Swenson, Crandall, Guy, Lenz, Ringwall, \& Walling, "Development of the RFD Linac Structure", Proc. of the 1995 Particle Accelerator Conference, Dallas, TX, 1995.

4. D.A. Swenson, F.W. Guy, K.R. Crandall, "Merits of the RFD Linac Structure for Proton and Light-Ion Acceleration Systems", Proc. of EPAC'96 Conference, Sitges, Spain, 1996.

5. D.A. Swenson, K.R. Crandall, F.W. Guy, J.M. Potter, T.A. Topolski, "Prototype of the RFD Linac Structure", 1996 Linac Conf., CERN, Geneva, Switzerland, 1996.

6. D.A. Swenson, "12-MeV Proton Linac for PET Isotope Production”, Institute of Clinical PET Conf., Orlando, FL, 1996.

7. D.A. Swenson, "CW RFD Linacs for the BNCT Application", Fourteenth Intern. Conference on the Applications of Accelerators in Research and Industry, Denton, TX, 1997.

8. D.A. Swenson, K.R. Crandall, F.W. Guy, J.W. Lenz, W.J. Starling, "First Performance of the RFD Linac Structure", Proc of 1998 Linac Conference, Chicago, IL, 1998.

9. D.A. Swenson, "Compact, Inexpensive, Epithermal Neutron Source for BNCT", Proceedings of CAARI'98, Denton, TX, 1998. 